Para citar este artículo / To cite this article:

Leclerc, Annie. «Une nouvelle affirmation du féminin à partir des années soixante-dix. Témoignage». En

Feminismo/s, 34 (diciembre 2019): 215-232. Dosier monográfico: Estado actual de la investigación en Literatura francesa y Género: balance y nuevas perspectivas / État présent de la recherche en Littérature française et Genre: bilan et nouvelles perspectives, coord. Ángeles Sirvent Ramos, DOI: 10.14198/fem.2019.34.10

\title{
UNE NOUVELLE AFFIRMATION DU FÉMININ À PARTIR DES ANNÉES SOIXANTE-DIX. TÉMOIGNAGE
}

\section{A NEW AFFIRMATION OF THE FEMININE FROM THE SEVENTIES ONWARDS. TESTIMONY}

\section{Résumé}

Annie Leclerc nous offre des réflexions personneles sur sa trajectoire vitale et intellectuelle vers la revendication de la différence et de la valeur et la puissance des femmes. Elle fait allusion dans sa conférence à sa passion pour la philosophie et l'écriture, à sa prise de conscience de l'infériorisation des femmes et l'utilisation qu'on en a fait pour la création du mythe de la supériorité masculine. Elle nous accorde le témoignage de sa prise de position et de sa contribution au mouvement des femmes dans la lutte pour la libération du désir et de la parole, ainsi que le témoignage de son éloignement des positions de Beauvoir. Loin de la lutte des sexes, Leclerc nous propose non pas conquérir mais partager; loin de toute sorte de guerre, de domination, elle montre le besoin d'une nouvelle écologie politique dans laquelle les femmes ont leur mot à dire.

Mots-clé: Annie Leclerc; écriture féminine; féminisme de la différence; écologie politique; Simone de Beauvoir.

\begin{abstract}
Annie Leclerc offers personal reflections on her vital and intellectual trajectory towards claiming difference and the value and power of women. In her lecture, she refers to her passion for philosophy and writing, her awareness of the inferiority of women and the use of it to create the myth of male superiority. She gives us a testimony of her position and contribution to the women's movement in the struggle for the liberation of desire and speech, as well as the testimony of her distance from Beauvoir's positions.
\end{abstract}

Los contenidos de la revista se publican bajo una licencia de Creative Commons Reconocimiento 4.0 Internacional (CC BY 4.0)

Feminismo/s 34, diciembre 2019, pp. 215-232 
Far from the struggle of the sexes, Leclerc proposes not to conquer but to share; far from any kind of war, from domination, she shows the need for a new political ecology in which women have their say.

Keywords: Annie Leclerc; Feminine writing; Feminism of difference; Political ecology; Simone de Beauvoir.

\title{
ANNIE LECLERC. LA JOUISSANCE DE VIVRE. IN MEMORIAM
}

\author{
Ángeles SIRVENT RAMOS \\ Coordinadora del dosier monográfico
}

Annie Leclerc nous a rendu visite en avril 2005. Je l'avais invitée à propos du $\mathrm{V}^{\mathrm{e}}$ Séminaire Femme-Littérature. J'adorais son écriture engagée mais douce, conciliatrice, que j'ai fait connaître à nos élèves. Je voulais que sa «parole de femme», intelligente, sensible, nous enveloppât dans les sessions du séminaire.

J'avais lu, parmi bien d'autres, sa Parole de femme, de même que la très belle préface de l'édition de 2001, que j'ai bien aimée, et qui se s'offrait comme un besoin encore d'éliminer toute forme de domination, de violence; une confirmation, autrement, et avec plus de conviction, des réflexions énoncées plus d'un quart de siècle avant: Le monde a besoin, et plus que jamais, des femmes. Le politique doit s'imbriquer avec l'écologie, la politique c'est l'écologie au sens le plus large, et avec les femmes.

Nous avions parlé avec les étudiants et les étudiantes des textes de Cixous, de Leclerc, comme nous continuons à le faire. Ils en avaient déjà lu certains d'entre eux, s'étaient introduits dans la pensée de la différence.

Annie Leclerc nous disait qu'il s'agirait de simples réflexions, de réflexions toutes personnelles, qu'elle ne prétendrait en aucune manière nous faire la leçon. Le public l'a adorée. Sa voix tranquille, sa sensibilité, ses propos sages, sa gentillesse a embaumé l'ambiance de la salle.

Elle m'a parlé de sa maladie. Elle attendait les résultats de certains examens médicaux, mais elle n'avait pas voulu rater son engagement avec nous. Et je la remercie infiniment de m'avoir permis de la connaître, après avoir

Feminismo/s 34, diciembre 2019, pp. 215-232 
goûté son écriture, d'avoir pu partager en intimité ces quelques journées, ces espaces alicantins.

À son retour elle m'avait écrit, les examens ne s'avéraient pas bons. Elle allait mourir un an plus tard, le 13 octobre 2006. J'ai partagé avec mes collègues la tristesse de sa disparition trop peu de temps après son départ d'Alicante. Elle nous avait laissé en cadeau, sa -je crois- dernière conférence.

Les mots d'Annie sont restés gardés pendant des années. Mais nous devons permettre de faire écouter sa voix, sa dernière voix que nous transcrivons maintenant sur le papier.

Faire renaître sa parole me renvoie à sa présence. Personne ne m'avait exprimé un tel souhait lors des différentes éditions du Séminaire. «Pourronsnous nager?» m'écrivit-elle quelques jours avant son arrivée à Alicante. Et nous avons nagé, en rappelant avec la caresse de l'eau son Éloge de la nage, «abandon à la seule jouissance d'exister», comme elle-même l'avait écrit.

Le plaisir de l'eau nous était commun, nous était nécessaire à l'une comme à l'autre, son frôlement doux, sa fraîcheur encore en ce mois d'avril, sa caresse en même temps. Nous partagions la même Méditerranée, celle d'Alicante, celle de ses vacances en Crète.

Elle m'avait parlé de certains textes inachevés, qui permettront la publication posthume de Paedophilia ou l'amour des enfants, grâce aux soins de sa bonne amie, l'écrivaine Nancy Huston; elle m'avait avoué son dernier projet: publier un volume «consacré à la fabrication de l'identité masculine autour de la guerre et du mythe de la supériorité masculine qui s'y associe». Elle envisageait d'examiner «les transformations radicales en cours concernant les hommes et les femmes (émancipation des femmes, contraception, éloignement du spectre de la guerre, un autre regard sur la violence, sur l'enfant...)», et dont le titre provisoire était La légende du sexe fort. Elle n'a pas pu l'accomplir.

Ce présent volume, qui prétend offrir un bilan des recherches sur la littérature et le genre, accueille les réflexions dernières d'Annie Leclerc, bilan personnel de l'écriture des femmes. Un simple «témoignage», comme elle avait voulu l'inscrire dans le titre. 


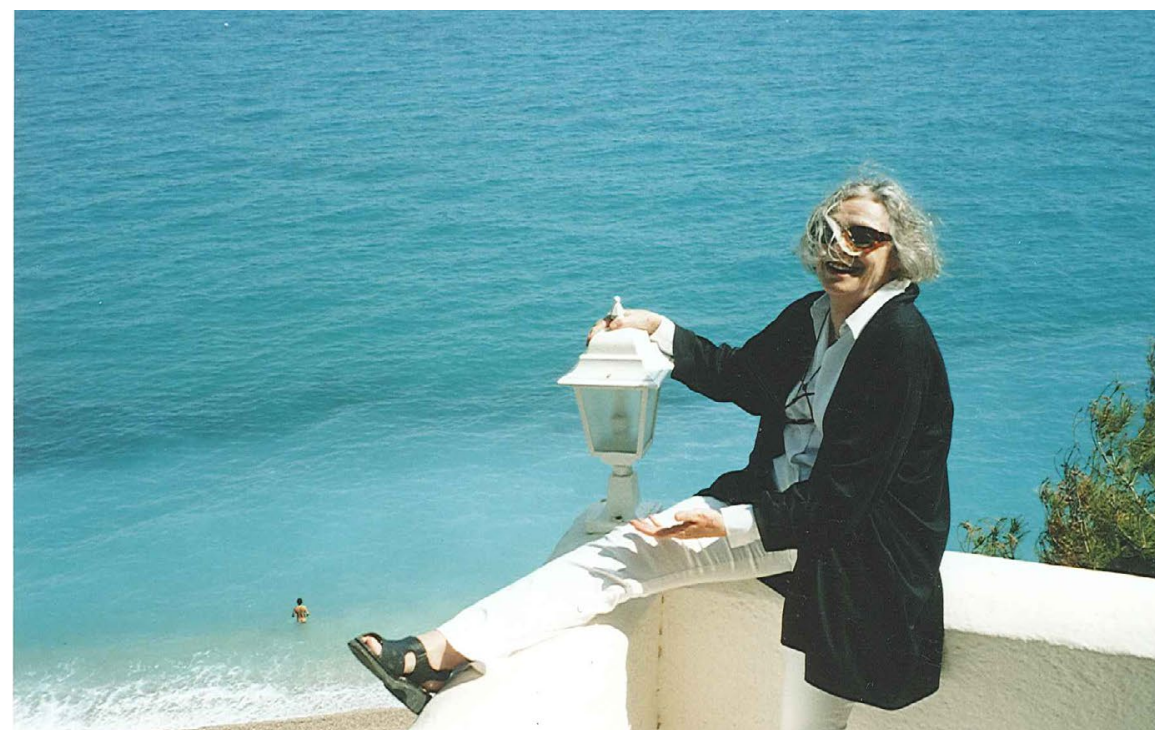

\section{UNE NOUVELLE AFFIRMATION DU FÉMININ À PARTIR DES ANNÉES SOIXANTE-DIX. TÉMOIGNAGE ${ }^{1}$}

Annie LECLERC
Philosophe. Écrivaine

Bonjour à tous. Ainsi me voilà parmi vous, l'unique femme qui ne soit pas universitaire, mais d'abord et depuis bien longtemps seulement écrivaine. Les enseignants ont naturellement vocation à la parole et à la transmission

1. Conférence offerte dans le Ve Séminaire Femme-Littérature: «La representación pública de lo privado: L'écriture de la différence des sexes et ses aventures». Universidad de Alicante, avril 2005.

Encore qu'Annie Leclerc nous avait laissé son texte, ses réflexions s'en libéraient à maintes reprises. C'est sa conférence, transcrite littéralement, qui s'offre ici, d'où le caractère oral qui traverse les lignes.

Enregistrement de la conférence par José Manuel Richart Uriendo en avril 2005; transcription par Mathilde Robin, novembre 2019.

Feminismo/s 34, diciembre 2019, pp. 215-232 
d'un savoir, mais les écrivains, je ne dispose d'aucun de ces savoirs qui se transmettent dans les universités. Je ne pourrai rien vous transmettre que le témoignage d'une femme particulièrement impliquée à travers l'écriture dans les questions qui vous intéressent. Mais comment vous présenter mon écriture? Je devine et je crains -c'est pourquoi je la devance- la question que vous ne manquerez sans doute pas de me poser, question déjà régulièrement posée aux nombreuses femmes qui dans les années soixante-dix se portaient ouvertement à l'écriture en tant que femme: celle de savoir si on pouvait concevoir une écriture spécifiquement féminine et en quoi elle consistait. Autant le dire tout de suite: je ne sais pas répondre à cette question. Je sais que je suis une femme, mais qu'est-ce qu'une femme, une vraie femme, une femme éternelle? Franchement, je ne sais pas. De même, je sais que j'écris, mais je suis incapable de définir à quel genre particulier d'écriture je me prête.

Romancière, poète, essayiste, j'avoue avoir été également sollicitée par une recherche de la vérité (comme on fait en philosophie ou dans l'ensemble des sciences humaines) et par le désir d'expression de mes émotions les plus intimes, comme autant de petits signes que nous envoie notre corps, non seulement pour intensifier notre jouissance à vivre, mais pour déchiffrer le sens que nous pouvons donner aux actes humains en général. Je me suis même laissé aller à écrire de la fiction, des textes qui m'ont souvent appris de moi des choses que j'ignorais, comme le font nos rêves.

À l'époque de la publication de Parole de femme, on m'a dit -pas toujours de façon très aimable- que c'était une manière bien féminine de procéder ainsi: ce mélange des genres, du corps, de l'esprit, de l'intime, du général, du lyrisme et d'une prétention à la rationalité ne pouvait que heurter les catégories bien définies des productions textuelles. Et qui est-ce que ça heurtait ? Les universitaires, les critiques littéraires, les libraires (qui ne savaient pas dans quel tiroir on allait me mettre), et particulièrement en France où on est toujours ardemment prié de ne pas mélanger les torchons et les serviettes -ça c'est une expression courante en France: surtout, ne mélangeons pas les torchons et les serviettes!. Quand on trouvait mon affaire trop embrouillée, moi, je la trouvais au contraire simplissime, coulant de source. Il me semblait que c'était comme ça qu'on pensait, engluée dans sa vie et ses expériences. Mais les expériences, ce sont les autres aussi.

Feminismo/s 34, diciembre 2019, pp. 215-232 
J'ai beaucoup lu. Les livres m'ont nourrie et ne cessent de me nourrir, livres de femmes, de philosophes, de poètes, d'historiens: impossible de savoir ce que j'aurais écrit et ce que je serais sans eux. Il me semble que si j'avais été universitaire -ça aurait pu arriver-, je n'aurais pas eu le temps de vagabonder de façon aussi anarchique en écriture et parmi les livres. Mais il me faut bien reconnaître que s'il n'y avait pas d'universitaires comme vous pour me prêter quelque attention de temps à autre, je n'existerais tout simplement pas. C'est pourquoi je vous remercie de tout cœur de m'avoir invitée et de m'écouter si gentiment. Je vous remercie aussi -ça, j'ajoute- ce que vous êtes, d'avoir une si belle université. Je suis vraiment éblouie et émerveillée de cette rencontre, que je n'oublierai pas.

Dès ma jeunesse, pour ne pas dire dès l'enfance, ce à quoi j'ai été le plus immédiatement sensible dans le partage des sexes c'est à la mise à l'écart des femmes dans tous les domaines de la création artistique et intellectuelle. Pourquoi, me disais-je, si peu de femmes philosophes, scientifiques, musiciennes, peintres? Qu'est-ce que c'est que cette histoire? Je n'ai jamais cru -vraiment jamais, ça, je vous le jure-ce qu'on essayait de me répondre alors: qu'elles n'étaient peut-être pas faites pour ça, qu'elles n'avaient sans doute pas les dons nécessaires. C'était comme si on voulait me consoler d'avance et m'inviter à renoncer à toute entreprise qui irait dans ce sens. Je savais que je ne renoncerais pas. Il restait à se demander pourquoi cela se passait ainsi. Pourquoi toujours cette infériorisation des femmes, cette mise sous tutelle, cette invitation à se taire? Lidée m'est vite venue que la déconsidération des femmes dans les domaines les plus prestigieux de l'activité humaine, c'està-dire le pouvoir et la pensée, servait à la valorisation du sexe masculin et à la fabrication du mythe de leur supériorité. La question alors qui se posait à moi était celle du pourquoi? J'ai d'abord pensé qu'il y avait quelque chose de trop puissant, de trop fort en vérité chez les femmes, dans le secret de la création, porteuse du mystère de la reproduction. Je pensais surtout à un désir des hommes de s'approprier une part de cette puissance, de la contrôler, de la dominer.

Or, moi, je rêvais de création, et pas seulement d'enfants. Très vite il m'est apparu que je voulais écrire, faire des livres, publier. Il faut dire que ce désir était rendu possible par un contexte général d'émancipation des filles, malgré la permanence des idées reçues qui ont toujours la vie plus longue

Feminismo/s 34, diciembre 2019, pp. 215-232 
que la réalité. Les jeunes filles de ma génération suivaient un même cursus scolaire que les garçons. On était en période de croissance économique et la perspective d'une réelle émancipation sexuelle se profilait. Laccès au monde du travail paraissait sans entrave. Ainsi, je n'avais ni l'envie de conserver ma virginité, au contraire, j'avais hâte de la perdre, d'être réellement une femme. Dans le monde traditionnel de la création, restait qu'on distinguait l'œuvre des hommes et les ouvrages de dames -c'est encore un peu vrai d'ailleurs. L'œuvre des hommes était censée nourrir la conscience humaine et le sens de son destin; les ouvrages de dames, le tricot, la cuisine, enfin toutes ces choses, chargeaient de servir, nourrir et agrémenter la vie quotidienne. Je n'avais rien contre les ouvrages de dames, mais ce à quoi je prétendais avant tout, c'est à l'œuvre.

Le mouvement des femmes des années soixante-dix s'origine dans un mouvement de révolte contre la dévalorisation générale des femmes, et il va se jouer essentiellement sur deux fronts: d'une part la dénonciation du mépris sexuel, social et culturel dont elles ont été victimes au sein même des groupes militants en soixante-huit, dans le contexte général d'une lutte pour la libération du désir, de la parole, de l'action. Ça, j'insiste, je fais une parenthèse: vraiment, le mouvement des femmes des années soixante-dix est né dans le mouvement de libération générale de soixante-huit, et les femmes se révoltaient contre leurs copains, contre leurs amis qui se comportaient mal avec elles: «les femmes, vous allez faire les tracts, mais vous ne vous occupez pas de prendre la parole en public». Donc, d'une part, ça: les luttes pour l'accès libre à la contraception, la reconnaissance de l'avortement, l'égalité salariale, le partage des tâches domestiques, ont été menées dans ce sens. Et les hommes me disaient d'ailleurs: «écoutez, les filles, ça, c'est secondaire, on verra plus tard; d'abord la révolution, et après on s'occupe de vous». Et nous, on disait: «Non, ensemble, on fait tout ensemble, les femmes et les hommes».

D'autre part, l'autre front: la volonté affirmée (en leur nom propre) de se prononcer sur les questions les plus graves de la société, dans le domaine de la connaissance, mais aussi sur la conduite même de la société; non pas seulement de mêler leurs voix à celles des hommes. Considérant qu'elles avaient leur mot à dire, elles se considérèrent comme une force politique autonome, d'où la prolifération de textes, journaux, prises de position (non sans luttes internes). Une part importante des femmes se levèrent contre le féminisme 
à l'ancienne -qu'elles considéraient à l'ancienne, comme inspiré seulement par le désir de partager le gâteau du pouvoir, des honneurs, de l'argent, le rejet des attributs de la féminité, l'aliénation donc à la phallocratie. Moi, je n'entrais pas dans ces combats parce que je pensais que, quelles que soient leurs divergences, toutes les femmes étaient d'accord pour lever une censure d'expression du féminin en général, dans la sexualité, la société, la politique, les sciences et les arts.

J'aimais être une fille, sans doute pour des raisons familiales et conjoncturelles particulières dans lesquelles je n'entre pas, et ce n'est pas dans un sursaut de révolte contre ma condition de femme que je me suis mise à l'écriture. C'est en suivant mon désir d'écrire et de philosopher que j'ai fini par rejoindre le mouvement des femmes qui s'était mis déjà en marche. Ou plutôt, le mouvement des femmes s'est emparé de Parole de femme et m'a emportée avec lui. La philosophie me passionnait: j'étais particulièrement sensible à la phénoménologie, appliquée à décrire l'intrication de notre être avec les phénomènes qui nous entourent et, partant, pour sa proximité avec la littérature. Je dénonçais la froideur philosophique, la séparation arbitraire du corps et de l'esprit qu'elle impliquait, sa prétention à la connaissance de l'homme universel abstrait. Je prétendais philosopher grâce à la raison et le concept, mais aussi à partir de l'expérience, de la sensibilité et des émotions. Le corps devait avoir une part imminente dans l'activité philosophique (en l'occurrence mon corps de femme) et il m'apparaissait que l'ensemble du corpus philosophique avait d'un même mouvement déconsidéré le corps et écarté les femmes des choses de l'esprit. Chantant le corps, et particulièrement le savoir que l'on pouvait tirer d'un corps féminin, c'est à l'esprit même de la philosophie que je m'en prenais.

Si le mouvement qui me conduisait à l'écriture de Parole de femme était plus philosophique que porté par une révolte de femme, il me conduisit à relever l'exclusion des femmes du système de représentation de l'Homme -avec un grand H- en général. Dans mon livre Parole de Femme, je parle beaucoup de l'homonymie de l'Homme en général et homme en particulier, c'est-àdire homme viril. Comment se fait-il que pour dire l'Homme en général, on dit quelque chose qu'on emploie surtout pour l'homme, l'homme viril. Par exemple, si on dit " un homme est un Homme » et « tout homme est un Homme », ça va, ça va bien; mais si on dit « toute femme est un Homme », cela

Feminismo/s 34, diciembre 2019, pp. 215-232 
fait rire, pourtant on pourrait pouvoir le dire. C'est pour cela que le titre Parole de Femme, qui a été traduit en espagnol par Palabra de mujer, est une ironie, et ne retrouve pas en espagnol l'ironie qu'il y a dans l'expression française, à cause de l'expression, en français, de « parole d'homme », qui est comme « parole d'honneur », qui veut dire « parole sérieuse ». Une femme ne peut pas dire «parole de femme », une femme n'a pas d'honneur, ce n'est pas sérieux, c'est un peu bizarre. C'est une histoire d'homme l'honneur, et l'expression « parole de femme » est un peu subversive. Et cette confusion entre Homme et homme permet de rendre manifeste le mécanisme du principe masculin érigé en loi universelle, comme si les hommes avaient directement accès à la vérité toujours, pour toujours.

Le propos de Parole de Femme fut donc de contester à un certain système de valeurs et de représentation du monde éminemment masculin: de la vie, de la sexualité (voisin de ce que Derrida à dénommé la phallogocentrie), articulé autour de la domination sur les autres, sur soi-même, sur le monde; la prétention métaphysique à atteindre le Vrai, à distinguer le corps de l'esprit, à définir le Bien et le Mal, le digne et l'indigne, le noble et l'ignoble. Ce qui s'éprouve et cherche à se dire à travers les épreuves du corps féminin (règles, grossesse, sexualité) ou du réel auquel les femmes sont confrontées (nourrir, secourir, réparer, éduquer) va dans un tout autre sens que la volonté de domination. D'autres valeurs moins spectaculaires, mais autrement plus favorables à la vie, à la jouissance et au partage s'y affirment. C'est alors l'ensemble du système idéologique au nom duquel les femmes ont été infériorisées qui est dénoncé. Il se trouve que cela rejoignait le sentiment d'une puissance féminine non encore exploitée, d'un apport inédit et fécond pour les temps à venir, et correspondait à l'esprit de ferveur joyeuse des manifestations des femmes d'alors. C'est ainsi que, solitaire et méditante dans mon coin, je me suis trouvée prise et emportée dans le flot du mouvement des femmes d'alors.

Alors, là, je fais une petite mise au point: en quoi cette approche se distinguait des lectures précédentes de la domination des femmes? Je prends pour exemple le mouvement qui a été très important autour du Deuxième sexe de Simone de Beauvoir. Simone de Beauvoir part dans son gros ouvrage, Le Deuxième sexe, de la condition socialement inférieure des femmes. Elle relève leur dépendance juridique, leur exclusion de la scène publique, leur statut d'éternelles soumises (à leurs pères, puis à leurs mari, puis finalement

Feminismo/s 34, diciembre 2019, pp. 215-232 
à leurs enfants). Elle dénonce surtout leur confinement aux tâches les plus basses, dévalorisantes et non-rémunérées de la société. Simone de Beauvoir propose à partir des notions sartriennes une interprétation de cette infériorisation: la femme, plus faible physiquement, affectée par les règles, alourdie par les grossesses est, par sa fonction de reproductrice, maintenue dans l'immanence (terme de la philosophie sartrienne, maintenue dans l'immanence, c'est-à-dire la force des choses, le réel, l'organique); la femme est éloignée de la transcendance, c'est-à-dire la capacité humaine à échapper aux déterminations naturelles ou conjoncturelles et à affirmer son être, qui n'est autre que la liberté. Là, je m'explique un petit peu: Simone de Beauvoir se coule dans le moule philosophique de Sartre, et pour Sartre, ce qui fait l'homme, c'est la liberté, c'est sa capacité à échapper à la nature, au déterminisme, etc. Simone de Beauvoir part du fait que les femmes sont prises dans la nature, qu'au fond, elles sont des sortes d'animaux. Elle le dit: il y a une animalité de la femme, ce qui n'est pas loin d'ailleurs d'une sorte de dégout pour le féminin.

Je vous dirai en toute vérité que je viens de relire le Deuxième sexe de Simone de Beauvoir -que j'ai bien connue par ailleurs et qui était une femme formidable- mais je trouve que le Deuxième sexe de Beauvoir est un livre remarquablement antiféministe. Pour moi, c'est une livre antiféministe, qui n'aime pas le féminin, qui n'aime pas le corps des femmes, qui n'aime pas la maternité, qui n'aime pas toutes ces choses dont elle pense qu'elles font le malheur des femmes. Elle ne remet nullement en question le système de représentation en place. La condition féminine est naturellement handicapée. Les hommes -comment elle explique donc que les hommes sont devenus plus forts?- c'est qu'ils sont plus forts, plus libres, plus indépendants physiquement, et qu'ils en ont naturellement profité. Alors, là aussi, il y a une croyance qu'il est naturel de profiter de sa force, que c'est quelque chose qui appartient à l'humanité. Je n'y crois pas. Elle dénonce le système de valorisation des femmes, articulé à leur devoir de reproductrice et censé les maintenir dans leur aliénation. Enfin, là -j'abrège un peu- le destin biologique des femmes, voilà ce qui, selon Simone de Beauvoir, entrave leur sort et explique leur maintien dans une condition inférieure.

Alors, je vais évoquer rapidement mon conflit avec Simone de Beauvoir, qui était avec qui j'ai été assez liée puisque j'ai travaillé dans l'équipe de la grande revue Les Temps Modernes (Sartre et Simone de Beauvoir avaient décidé

Feminismo/s 34, diciembre 2019, pp. 215-232 
de prendre des gens plus jeunes avec eux -car j'ai été beaucoup plus jeune que je le suis), mais au moment de Parole de Femme, il y a eu un conflit. Elle n'acceptait pas ma première remise en cause d'un être masculin censé incarner l'universel. Elle disait: «mais le Vrai est le Vrai, que ce soient les hommes ou les femmes qui le décident; un point c'est tout; pour tout le monde pareil». Que ce soient les hommes qui se soient prononcés sur les choses les plus importantes de la vie humaine ne changeait rien à l'affaire. La confusion en français entre Homme (au sens générique) et homme (au sens d'être masculin) ne lui semblait pas particulièrement intéressante.

Ma seconde observation était déjà plus grave: je disais que la «virilité» est le fruit d'un dressage particulièrement contraignant. Et cela pouvait passer pour une véritable attaque contre sa façon de lire l'oppression des femmes: la formule la plus célèbre de Simone de Beauvoir, ouvrant le deuxième volume du Deuxième sexe, c'est «on ne naît pas femme, on le devient». Or, moi, je trouvais que cette formule valait encore plus pour l'homme: "on ne naît pas homme (au sens de mâle, viril, être masculin), on le devient». C'est un montage social particulièrement contraignant, et même en un certain sens, si on juge d'un point de vue autre -que j'appelais féminin-, c'est un dressage inhumanisant: les hommes sont dressés à être durs, à refouler leurs larmes, etc. Je ne m'étendrai pas là-dessus pour le moment. Et à l'inverse alors, tout à fait à l'inverse de Simone de Beauvoir, je chantais les jouissances du corps féminin: la grossesse, l'accouchement, l'allaitement, que, elle, concevait comme des fardeaux, des chaînes, pour ne pas dire des fatalités dégoûtantes. Toutes ces choses, à moi, m'apparaissaient comme des expériences privilégiées de la vie, où le sens même de l'humain et de ce qui peut en faire le prix se découvrent.

Dernier point, beaucoup plus important que ce qu'il n'y paraissait à première vue et qui a donné lieu à beaucoup de querelles: j'avais examiné la déconsidération attachée aux tâches domestiques, et au nom de quelles autres valeurs elles étaient méprisées. Loin de dénigrer les tâches domestiques, je leur trouvais beaucoup de sens et d'humanité. Elles ne devenaient pénibles que parce qu'elles ne comptaient pour rien et qu'elles envahissaient la vie des femmes au point de les priver de tout le reste. Alors ça, c'est un point qui a l'air secondaire, l'histoire des tâches domestiques, mais pour moi, c'est très important. Trouver dégoûtant de laver, de nettoyer les carreaux, de faire la cuisine, de repasser, de faire le ménage, ce n'est pas ça, ce n'est pas du tout

Feminismo/s 34, diciembre 2019, pp. 215-232 
dégoûtant! Au contraire, c'est très noble. Le malheur vient de ce qu'on en fait trop, qu'on n'est pas payées pour ça, qu'on ne reçoit rien, qu'on nous méprise et qu'on n'a pas le temps de faire autre chose. La réplique n'a pas manqué et s'en est suivie du côté des Temps Modernes, la revue même de Sartre et de Simone de Beauvoir à laquelle j'avais contribué plusieurs années. Je rejoignais -on m'a dit que je rejoignais- les valeurs les plus traditionnelles de la féminité, que je chantais les joies possibles de la féminité (biologiques ou culturelles), que je tendais à maintenir les femmes dans leur condition de «servantes». Et on me disait -il y a eu un très gros article dans les Temps Modernes, un gros pavé-que je ne faisais que décliner le mythe bourgeois de la femme éternelle.

À vrai dire, j'ai été peu touchée par ces critiques, ni par les affrontements parfois très violents de courants distincts du féminisme luttant pour l'émancipation des femmes. Je pensais que toutes les femmes voulaient se libérer d'une tutelle millénaire et que la dénonciation des oppressions, des inégalités et du mépris était commune à toutes les femmes. Je voyais bien que les conflits entre les femmes étaient superficiels par rapport à l'ampleur d'un mouvement de fond, dans lequel les unes et les autres étaient emportées et ne cesseraient de l'être. Je crois que le mouvement des femmes commence à peine, ne fait que commencer et qu'il va aller très, très loin.

Je vais laisser ma parenthèse épistémologique là, une parenthèse où j'essaie d'expliquer que dans ma philosophie à moi, je ne crois pas à la guerre. Je crois qu'au fond les gens sont très près d'être d'accord les uns les autres, qu'ils ont l'air de se disputer, mais qu'au fond ils sont faits de la même chose, qu'ils pensent à peu près la même chose. Donc, il ne faut donc pas trop entrer dans les querelles, ce n'est pas intéressant. Bon, je n'entre pas dans cette parenthèse.

Alors, je crois qu'il faut considérer ensemble la montée en puissance des femmes tout au cours du vingtième siècle, leur accès au monde du travail, la scolarisation des filles, l'école publique et obligatoire, l'accès au droit de vote, la participation des femmes aux luttes de subversion politique, l'élaboration scientifique de méthodes contraceptives et les idéologies de la «libération». Je veux dire par là que, si j'ai fait quelque chose, je l'ai fait aussi parce qu'il y a un mouvement historique, et ce mouvement est très fort; l'apparition d'un véritable champ d'expression des femmes dans les arts, la littérature, les mouvements sociaux. Alors, moi, je fais remonter la naissance de la puissance des femmes (c'est un peu différent sans doute pour le contexte socio-historique

Feminismo/s 34, diciembre 2019, pp. 215-232 
espagnol) au moment de la Grande Guerre, la Guerre de 14-18, qui a été tellement terrible, cette guerre, qui a tellement décimé les hommes, qui les a tellement humiliés, que le sens même de la virilité guerrière a été atteint. Les hommes ont commencé à ne plus avoir envie d'être des hommes comme on leur avait appris à être des hommes.

Alors, peut-être pour vous les espagnols, les choses se sont peut-être passées autrement: le fascisme, la résistance aux dictatures fascistes, que l'on retrouve dans le culte du héros chez Malraux, par exemple, que je n'aime pas. Je n'aime pas Malraux, je pense que Malraux est un écrivain de la tradition de la virilité qui est en perte de puissance. Je n'ai jamais aimé Malraux. Je crois même que déjà dans Parole de Femme je m'en prends au héros selon Malraux. Je ne l'aimais pas parce que les hommes de ma génération, mes compagnons, mes camarades, vouaient une adoration pour Malraux, et ce Malraux m'énervait; mais comme m'énervaient les hommes de ma génération-que j'aimais par ailleurs, mais qui étaient des militants, on disait des «militants»... Et dans les années soixante-huit, on disait, ils disaient «le pouvoir est au bout du fusil». C'était une phrase de Mao, Mao Tsé-toung. Et moi, je ne voulais pas du tout d'un pouvoir au bout du fusil. Je vous fais remarquer combien le culte du militantisme est en net recul dans nos sociétés -et c'est tant mieuxdepuis l'effondrement de l'Union Soviétique et la chute du mur de Berlin.

Alors, je pense que tous ces différents mouvements qui se sont passés là autour des années soixante-huit, soixante-dix, doivent être mis en valeur. Je crois pouvoir souligner que les hommes et les femmes sont bien plus profondément d'accord qu'il n'y paraît (ça retrouve la parenthèse que j'ai laissée de côté): l'idée d'une guerre des sexes au sens strict du terme est pour moi une aberration. Il y a des résistances là où les privilèges sont menacés, où l'inquiétude identitaire gagne, mais c'est forcément transitoire. Il y a des révoltes, des violences, mais pas de guerre. Ça n'existe pas la guerre des sexes. Il y a des arrangements troubles, mais pas de réel divorce. Les hommes et les femmes sont condamnés à vivre ensemble. J'ai peut-être été plus sensible à ça encore dans mon livre Toi, Pénélope, qui est un livre qui raconte des histoires d'hommes: l'Odyssée raconte l'histoire d'Ulysse qui se combat avec des hommes, et l'on voit tout ça vu du regard de Pénélope qui voit ça un peu autrement, mais sans qu'il y ait un véritable divorce.

Feminismo/s 34, diciembre 2019, pp. 215-232 
Les années soixante-dix ont été marquées dans leur ensemble par une sensibilisation à d'autres valeurs que celles de la lutte. Je parlais tout à l'heure du militantisme, mais l'esprit était aussi tout à fait autrement: le vrai slogan, c'était «faisons l'amour, pas la guerre»; la jouissance, pas la conquête; le partage, pas la concurrence. Il y avait dans ces années la recherche d'un art de vivre plus tendre, moins dépendant des contraintes sociales et de la réussite professionnelle; un rapprochement plus fraternel des sexes sans doute favorisé par la mixité scolaire. Il s'est dégagé de ces années-là une sensibilité plus affirmée aux enfants, un assouplissement dans la distribution des rôles parentaux. Je crois pouvoir dire que le patriarcat est en net recul (ce qui ne va pas sans crises, sans craintes, sans menaces, bien sûr).

Nous sommes dans un autre contexte politique, le monde est dans un autre contexte politique: nous sommes sortis du colonialisme; le nationalisme s'affaiblit (et c'est une des raisons pour lesquelles je crois à l'Europe, je crois qu'il y a dans l'Europe un affaiblissement des nationalismes, forcément). Et puis, il y a l'autre contexte politique: c'est l'humiliation et la méfiance relatives aux grandes idéologies. On a vraiment souffert des grandes idéologies et on se tient à l'écart. D'un autre côté, montent dans le monde de grandes peurs nouvelles: l'avenir même de l'humanité semble compromis, une crainte de fond pour les générations nouvelles apparaît. C'est vrai que les jeunes ont peur de faire des enfants; ils en ont envie et ils en ont peur. C'est légitime. Lexpansion démographique vertigineuse est confrontée à la raréfaction des ressources, la dégradation de l'environnement, la prolifération des armes de destruction massive, l'accroissement des inégalités, le déferlement du terrorisme. Les années soixante-dix ont vu la naissance de l'écologie politique.

Ce mouvement -à mes yeux encore à peine débutant, mais destiné à se substituer progressivement aux politiques traditionnelles, qui étaient déterminées par l'affrontement des rapports de force-, cette écologie politique me semble très proche du féminisme nouveau. L'humanité va être impérieusement appelée à se retirer de la guerre et à aménager quelque chose comme la paix. Les femmes, depuis des siècles sinon depuis des millénaires, sont mieux préparées à la gestion du quotidien et du réel, à la réparation et au soin. Les femmes seront éminemment requises pour cette tâche. Les femmes, mieux disposées aussi par le phénomène de la gestation à la fabrication de l'autre en soi, ont une conscience de soi moins arrêtée aux bornes de l'existence

Feminismo/s 34, diciembre 2019, pp. 215-232 
individuelle. Je sens bien que j'ai commencé avant moi, que je continuerai après moi: je suis de passage. Passante et passeuse. Et ça ne me rend pas triste, au contraire, ça me rend active. Que cette conscience se répande avec la pénétration massive de la sphère publique par les femmes ne peut que donner une chance à l'humanité qui en a bien besoin. Ils ne se représentent peut-être pas tout à fait encore combien ils ont besoin des femmes, mais ça va venir.

Le vingtième siècle a déjà eu de très grandes penseuses qui commencent à peine à être lues. Je pense à Hannah Arendt et à Simone Weil, qui l'une et l'autre sont entrées dans des questions spécifiquement réservées aux hommes: le pouvoir, le travail, les luttes sociales, le sens des conflits humains... Et elles ont eu une façon très originale d'aborder ces questions dont on n'a pas encore tiré tout le profit qu'on en tirera plus tard -j'en suis sûre. De grandes artistes aussi, encore trop souvent écartées de la reconnaissance médiatique qui se porte toujours plus volontiers sur les hommes. Ça, c'est vrai, ça, c'est sûr: il y a autant de livres de femmes que d'hommes qui paraissent, et quand on calcule, il y a un dixième de comptes-rendus qui sont faits des livres de femmes. J'y fais attention car évidemment ces questions m'intéressent. C'est impressionnant. On peut noter la présence de plus en plus manifeste des femmes dans les arts qui sont plus attachés à la reconnaissance des œuvres que des auteurs. Par exemple, on voit peu de noms de femmes; les noms des femmes en littérature et en philosophie n'apparaissent pas. Mais on trouve beaucoup de femmes dans les domaines où on fait moins attention aux noms des auteurs, comme le cinéma, le théâtre et la composition musicale. Il y a énormément de femmes -je ne sais pas si c'est le cas en Espagne-il y a énormément de femmes qui font du cinéma.

Alors, je reviens à ma vision des choses et à la façon dont j'ai conduit mon écriture (puisque j'essaie de faire un témoignage). Je considère que tous mes travaux ont été menés à partir de ma position de femme, mais, qu'en même temps, mes travaux étaient soutenus par les grands travaux de pensée de mon temps, mais aussi éclairés par les conflits et les écueils auxquels la pensée de mon temps a été confrontée. Les deux grands soucis -j'essaie d'énoncer les deux grands soucis qui ont animé mon travail-: la jouissance de vivre dont j'ai toujours compris qu'elle ne pouvait se séparer de la transmission, du donner-recevoir; et, deuxième point, la question de la violence, dont il m'est apparu qu'elle survenait quand la transmission était empêchée, quand

Feminismo/s 34, diciembre 2019, pp. 215-232 
je me trouvais enfermée en moi, impuissante à donner, recevoir, prisonnière, étouffée.

Alors, pour moi, la violence est toujours associée à de la faiblesse. La violence est l'expression non pas d'une force, mais d'une impuissance: c'est quand les forces de la vie, de l'énergie, de la pensée, de la générosité, ne peuvent plus passer, que l'on devient violent. C'est une question pour moi essentielle qui anime tout mon travail actuel. Si par exemple les hommes sont violents, c'est parce qu'ils ne sont pas bien, c'est parce que ça ne va pas, c'est parce qu'ils n'arrivent pas à exister et à jouir de vivre comme ils pourraient et devraient jouir de vivre. Mon écriture devait non seulement interroger la jouissance, mais en favoriser l'approche; pas seulement analyser la violence, mais travailler à la dissoudre en moi, et peut-être au-delà, en mon lecteur. Bon, alors, je travaille à répandre la jouissance et aussi dissoudre la violence, la dissoudre aussi en moi, car elle est en moi, la violence, elle est en tout le monde. Et en travaillant à la dissoudre en moi, la dissoudre aussi en mon lecteur. J'ai été guidée par la recherche d'une écriture authentique fondée dans mon expérience propre (moi-je, telle femme, telle histoire, telles rencontres...), non par narcissisme -enfin, je le crois-, mais par le secret d'exprimer une intimité susceptible de rejoindre d'autres intimités et de susciter leur propre expression dans un sens qui leur soit favorable. C'est d'ailleurs pour ça que je privilégie la littérature sur la théorie ou la simple parole.

Je me suis également beaucoup inspirée de l'amour de l'enfant, que je ne voudrais pas identifier au féminin, au maternel, parce que je crois que les hommes y sont tout autant sensibles, quoi que détournés par d'autres tâches. Je crois que les hommes -ça, j'en suis sûre- aiment autant les enfants, les petits-enfants, les toucher, les prendre et jouer avec eux, que les femmes. C'est pour ça que je dis que les hommes ont été dressés à ne pas faire un certain nombre de choses qu'ils ont très envie de faire. En ce sens, la pensée de Nietzsche aura été -me semble-t-il- le dieu caché de mon inspiration. Nietzsche est un penseur très compliqué, mais je trouve qu'il est très féminin en ce sens. Je le cite: «être enfant, voir l'enfant, celui qui n'est pas nous, mais au-devant de nous»; et là, une autre citation de Nietzsche: «l'enfant, innocence et oubli, un renouveau et un jeu, une roue qui roule d'elle-même, un premier mouvement, une sainte affirmation». J'ai formé petit à petit, dans cette inspiration, tout un système de lecture de l'humain, de la différenciation

Feminismo/s 34, diciembre 2019, pp. 215-232 
des sexes, de l'histoire même de ce partage. On peut dire que c'est extrêmement prétentieux, mais tout autant très modeste. Je n'ignore pas que c'est l'élaboration de ce qui allait dans le sens de mon désir, de ma sensibilité, de ce qui était susceptible de consoler mes peines et d'augmenter mon bonheur de vivre, par une certaine représentation de l'humain, du partage des sexes et du sens que nous serons susceptibles de donner à la poursuite de la vie.

Ainsi, mon dernier travail en cours s'attache à rendre manifeste la fabrication de la «virilité» et la mise en place symbolique (garantie par les institutions) d'une prétendue supériorité masculine autour du fait guerrier -autant vous dire que je ne crois pas à la supériorité masculine, évidemment. Je m'attache à montrer le recul de la guerre en tant que telle, et partant la nécessité de fabriquer des êtres capables de la chose la plus extravagante du monde, qui est de se mettre en disposition de tuer des hommes inconnus qui ne vous ont rien fait ou de s'exposer soi-même à la guerre. Je crois que c'est parce que l'humanité a eu besoin, vraiment la nécessité pour survivre, de faire la guerre, de tuer, que nous avons fabriqué des hommes comme ils ont été fabriqués.

Mais je crois aussi que la guerre s'en va: on ne peut plus faire la guerre. Si peu à peu les nations se défont, pas pour des raisons morales, mais sous la poussée de l'internationalisation des échanges; si les guerres frontales à l'ancienne deviennent de plus en plus improbables et interdites par le nucléaire lui-même; si l'urgence première n'est plus de gagner sur l'adversaire, mais de sauver la Terre et son avenir; la virilité perd de son éclat et de sa nécessité. Les peurs nouvelles qui se font jour, loin de surenchérir dans l'armement, comme nous l'avons fait pendant des générations, nous acculera au désarmement (tâche longue, délicate, sans éclat, mais seulement inspirée par la poursuite de la vie de chacun). Pourvu que les enfants grandissent, trouvent à se nourrir, à s'épanouir, sans se battre, sans se jalouser, et à transmettre aux suivants une très modeste ambition de mère à laquelle il faudra bien que les hommes se rendent, l'humanité va devoir se référer à d'autres valeurs qu'à la conquête et à la domination. Je ne fais là que poursuivre un mouvement engagé dans mon écriture depuis plus de trente ans.

Alors bon, je pense que c'est un système de lecture qui a commencé à naître en moi quand j'étais enceinte. Je pense que le sens qu'il faut transmettre -il y a quelque chose à transmettre- est là, la vie, le monde est menacé. Donc, il va falloir qu'on s'occupe de cela. La position des femmes dans la

Feminismo/s 34, diciembre 2019, pp. 215-232 
transmission de la vie, la continuité d'un lien de chair entre la génération précédente et la suivante les rend plus aptes à l'appréhension d'une vérité forcément transitoire- que le souci de réussite individuelle, de performance, de gloire a trop souvent dérobée aux hommes. Les femmes savent mieux que les hommes que nous sommes ici de passage. Rien ne vaut que ce que nous savons recueillir de meilleur du passé, faire fructifier au présent et transmettre à ceux qui suivent. À travers elles, la mort fait moins peur, la soif de toute-puissance s'apaise. L'art, la création, l'écriture, n'ont plus pour but la célébration des auteurs des œuvres dans un vaste système de compétition -donc de guerre-, mais la « culture » de l'humain au sens vrai du terme, et au temps où les menaces les plus inquiétantes se font jour. C'est la modeste application à prolonger son existence en l'incitant à se délivrer de ses maux les plus effrayants: la misère, l'injustice et la violence. La beauté n'est rien que la vibration de ce désir toujours renouvelé.

Je vous remercie. 